\title{
Project 86800
}

This report summarizes work after fifteen months of a three-year project. The project is part of a larger effort to improve barrier properties of any polymer by 10,000 times. This implies that any polymer barrier now good for a day will be made effective for a decade. We use two strategies to achieve these improvements. The first, primarily supported by the Air Force Office of Scientific Research, uses aligned impermeable flakes of mica, clay, and other materials to enhance barrier properties 10 to 100 times. The barrier works against all diffusing species. This strategy, which we are applying to make corrosion resistant paints, works both in the unsteady state and in the steady state. Our second strategy, supported by this grant, uses reagents within the barrier polymer to neutralize specific species. This strategy works only in the unsteady state, while the reagents last. It is extremely effective, however, sometimes achieving increases in barrier properties of 1000 times or more. In the paragraphs below, we give results for both strategies. Acid Barriers Initial studies were performed with acid as a model contaminant, zinc oxide as the reactive material, and inclusions of mica or clay flakes. Permeability reductions of 10 to 4000 times were observed in flake-filled composite membranes. The reduced permeability can occur because of chemically reactive groups in the membrane and because of mica or clay flakes aligned parallel to the membrane surface which increase the diffusion path length. The inclusion of zinc oxide increased the time to permeate across the film by as much as 3000 -fold. Mica flakes reduce both the permeation time and the leak rate by as much as 100 times. Both of these effects can be predicted theoretically without adjustable parameters. We have also shown that these effects can be combined to yield still better barriers. Contaminant Barriers A. $\mathrm{Fe}(0) / \mathrm{PVA}$ membranes Polyvinyl alcohol (PVA) membranes containing zero-valent iron $(\mathrm{Fe}(0))$ particles were developed and tested as model barriers for a variety of environmental contaminants. While PVA is a poor choice of barrier material, it is good for initial laboratory experiments because it is easy to synthesize and has a high permeability which allows quick measurement of breakthrough lag time. $\mathrm{Fe}(0)$ was selected as the immobilized reactant, for $\mathrm{Fe}(0)$ is known to reductively transform oxidized contaminants such as chlorinated solvents, nitroaromatic compounds, and heavy metal ions. Carbon tetrachloride ( $\mathrm{CCl} 4)$, cupric ion $(\mathrm{Cu} 2+)$, nitrobenzene, 4-nitroacetophenone, and chromate ( $\mathrm{CrO} 42-)$ were selected as model contaminants. The theory for diffusion through a membrane without reaction predicts that the downstream concentration changes according to the following equation at the beginning of the breakthrough: $\mathrm{Cdown} / \mathrm{Cup}=(\mathrm{PA} / \mathrm{LV})(\mathrm{t}-\mathrm{tlag})$ where tlag $=(1 / 6 \mathrm{D}) \mathrm{L}^{\wedge} 2(1)$ where Cdown and Cup are the contaminant concentrations in the downstream and upstream cells, $\mathrm{D}$ is the diffusion coefficient for the selected contaminant in the membrane, $\mathrm{P}$ is permeability of the membrane for the contaminant $(\mathrm{P}=\mathrm{HD}$ where $\mathrm{H}$ is a membrane-water partition coefficient for the contaminant into the membrane), $\mathrm{L}$ is the membrane thickness, $\mathrm{V}$ is the volume of the downstream cell, $\mathrm{A}$ is the cross-sectional area of the membrane available for diffusion, and $t$ and tlag are time and breakthrough lag time, respectively. This theory has been widely verified. For a membrane containing a reactive material that consumes contaminants via an irreversible reaction, the same form of equation holds, but a new expression for the lag time is needed: tlag $=(1 / 6 \mathrm{D}) \mathrm{L}^{\wedge} 2 *(3 \mathrm{Co} / \mathrm{vHCup})(2)$ where $\mathrm{v}$ is a stoichiometric coefficient of the reaction and Co is the initial concentration of reactive material in the membrane. In this work, $v$ is 1 for carbon tetrachloride (i.e. the major product in a short experiment is chloroform), 1 for $\mathrm{Cu} 2+, 3$ for nitrobenzene and 4nitroacetophenone, and 1.5 for $\mathrm{CrO} 42-$. In equation (2), Co is the initial concentration of the reactive material (e.g., $\mathrm{Fe}(0)$ in this study) in the membrane. This equation, which assumes a constant Cup and an instantaneous reaction (i.e. the reaction is much faster than diffusion through the membrane), has been verified for acid-base reactions. Breakthrough curves of carbon tetrachloride, $\mathrm{Cu} 2+$, nitrobenzene, 4nitroacetophenone, and $\mathrm{CrO} 42-$ through pure PVA and $\mathrm{Fe}(0) / \mathrm{PVA}$ membranes were collected. The lag times without reaction are less than two minutes. The lag times with reactions are much larger. A clear increase in the breakthrough lag time was found for carbon tetrachloride and $\mathrm{Cu} 2+$. The lag times for $\mathrm{Fe}(0) / \mathrm{PVA}$ membranes against carbon tetrachloride and $\mathrm{Cu} 2+$ are $352 \mathrm{~min}$ and $374 \mathrm{~min}$, respectively, while the corresponding lag times for PVA membranes are respectively $1.4 \mathrm{~min}$ and $1.0 \mathrm{~min}$ (after adjustments). The similar slopes of the breakthrough curves for PVA and $\mathrm{Fe}(0) / \mathrm{PVA}$ revealed that the permeability was not greatly affected by the presence of $\mathrm{Fe}(0)$. The breakthrough lag time for the $\mathrm{Fe}(0) / \mathrm{PVA}$ membrane against nitrobenzene is $53 \mathrm{~min}$, while it is $0.45 \mathrm{~min}$ for the pure PVA membrane (again after adjustments). There are two obvious reasons for the smaller effect. First, nitrobenzene requires more $\mathrm{Fe}(0)$ for reduction $(\mathrm{v}=3)$ compared with carbon tetrachloride and $\mathrm{Cu} 2+(\mathrm{v}=1)$. Second, the permeability of the membrane to 
nitrobenzene is higher than for the other two compounds, which also decreases the lag time (see eq 2). Surprisingly, the lag times in reactive membranes are predicted to be independent of reaction kinetics. To test this prediction, 4-nitroacetophenone was selected as a model contaminant. 4-Nitroacetophenone reacts faster with $\mathrm{Fe}(0)$ than nitrobenzene does. The breakthrough lag time for 4-nitroacetophenone diffusing across the $\mathrm{Fe}(0) / \mathrm{PVA}$ membrane is $46 \mathrm{~min}$, close to that for nitrobenzene through a similar $\mathrm{Fe}(0) / \mathrm{PVA}$ membrane and thus consistent with the prediction. The lag for 4-nitroacetophenone is 0.65 min for the pure PVA membrane (after adjustments). The breakthrough for CrO42- is affected less by the iron incorporation. The breakthrough lag time for the pure PVA membrane is 7 min (after adjustments), while it is $87 \mathrm{~min}$ for the $\mathrm{Fe}(0) / \mathrm{PVA}$ membrane. Batch experiments show that CrO42- reacts very slowly when its concentration is high (e.g., > 1000 micromolar), probably because of deposition of $\mathrm{Cr} 2 \mathrm{O} 3$ on the iron surface. The large lag time for the pure PVA membrane, which is reproducible, may reflect reaction of $\mathrm{CrO} 42-$ with the PVA itself to form an ester. Eq 2 can predict the diffusion lag time for the $\mathrm{Fe}(0) / \mathrm{PVA}$ membranes by assuming that all of the iron in the membrane is consumed before the breakthrough. The required parameters ( $\mathrm{L}, \mathrm{C} 0, \mathrm{Cup}, \mathrm{v})$ are all known from the experiments, and $\mathrm{P}$ is obtained from a diffusion experiment using a pure PVA membrane. The theory does predict increases in lag times of 100 or more times, which are observed. The increases measured, however, are less than predicted. The ratio of the predicted lag time to the corresponding experimental value is smallest for $\mathrm{Cu} 2+(\sim 2)$, larger for carbon tetrachloride, nitrobenzene, and 4-nitroacetophenone $(\sim 6)$, and largest for CrO42- $(\sim 13)$. We believe that theory and experiment disagree because not all of the reactive material in the membrane is consumed by the contaminant. In particular, only $44.7 \%$ of the iron (based on the dry membrane prior to hydration) is used for the reaction with $\mathrm{Cu} 2+$ before the breakthrough and $19.0 \%$ for carbon tetrachloride. The same analysis show that $24.5 \%, 16.7 \%$, and $6.0 \%$ of the iron is available for nitrobenzene, 4-nitroacetophenone, and CrO42-, respectively. These numbers do not account for iron loss during hydration of the PVA membrane $(\sim 50 \%)$. Thus, of the iron remaining in the membrane after hydration, $89 \%$ is used by $\mathrm{Cu} 2+$ and $38 \%$ by carbon tetrachloride. We hypothesize that the remaining iron is still contained within the membrane and coated with an iron hydroxide which slows the reaction to a point where contaminants can diffuse across the film before they react with the reactive particles. B. Fe(0)/HDPE membranes A method to manufacture zero-valent iron bearing high-density polyethylene (HDPE) membranes has been developed. Polyethylene beads are melted in a batch mixer and a known mass of iron metal of the desired particle diameter is added to the melted HDPE. Upon cooling, the mixture is scraped out of the mixing chamber. The Fe(0)/HDPE mixture is then placed on a hot press on which membranes of the desired thickness are pressed. We have made membranes varying in thickness from 80-800 microns, with iron loadings ranging from 5-20\% by mass, and with iron particles with diameters of $100 \mathrm{~nm}, 10$ microns, 50 microns, and 150 microns. Use of the HDPE also required a minor modification of the diaphragm cell testing apparatus because a seal between the HDPE membrane and the glass diffusion cells could not be kept water tight. Thus, a ring of PVA is used as a gasket to provide a seal. Preliminary experiments with carbon tetrachloride as the contaminant have shown that the iron bearing HDPE membranes have longer lag times than pure HDPE. To date, results are not quantitative as we had difficulty maintaining a constant concentration of carbon tetrachloride in the upstream cell. We have now overcome this problem by having the carbon tetrachloride in the upstream cell continuously replenished with a saturated carbon tetrachloride solution via a recirculation system. Modeling A numerical model for a system of spherical, uniformly distributed reactive particles within a barrier membrane was also developed. It is assumed that the particles shrink uniformly as the reaction occurs. The key parameters are the ratio of reaction rate versus diffusion rate (the Damkohler number), particle loading, and membrane thickness. The coupled, non-linear partial differential equations are solved using a centered finite difference method in space and the explicit Euler method in time. The model suggests two membrane performance parameters which quantify the solute flux after contaminant breakthrough: the leakage and the kill time. The kill time is the time at which the total amount of solute that has passed through the barrier is deemed unacceptable. The leakage quantifies not only how much contaminant leaks through, but also how long it is there. We have successfully evaluated the leakage and kill time as a function of particle loading and reaction rate. 